\title{
LEY 7/1994, de 5 de diciembre, de la Generalitat Valenciana, de la Infancia
}

\section{Preámbulo}

1. La defensa, protección y prevención de los riesgos de la infancia en una sociedad madura y democráticamente avanzada es un capítulo esencial en las políticas de bienestar social y en la consecución de una auténtica igualdad de oportunidades. Ya no es posible, sin atentar contra la cohesión social, que determinadas condiciones económicas, ambientales y familiares aparten a algunos niños de los beneficios colectivos y de las oportunidades sociales.

El principio de integración escolar, que consagró la Ley Orgánica 8/1985, de 3 de julio, del Derecho a la Educación, ha supuesto un auténtico cambio tanto en las prácticas educativas como en la concepción de la escuela ordinaria que asumió el reto de atender a alumnos y alumnas que demandan una respuesta diferencial. Los niños con necesidades especiales que anteriormente eran encaminados hacia otro tipo de centros se incorporan de este modo al sistema educativo ordinario.

El principio de universalización de la sanidad, que consagró la Ley 14/1986, de 25 de abril, General de Sanidad ha significado igualmente un salto cualitativo en la política tradicional de atención a las familias más vulnerables. La desprotección por razones de salud pública, que afectaban fundamentalmente a colectivos marginales, es atendido básicamente por el sistema ordinario de salud.

El principio de normalización de la asistencia social, que consagró la Ley 5/1989, de 6 de julio, de Servicios Sociales de la Comunidad Valenciana, está transformando igualmente la protección social a la infancia y a los colectivos socialmente marginados incorporándola a los sistemas ordinarios de atención a las necesidades y de promoción de la calidad de vida de los ciudadanos. Los niños con un ambiente familiar desestructurado o en situación socio-cultural desfavorecido están siendo atendidos por los servicios públicos abiertos a toda la población.Y la sociedad, ya concienciada de sus deberes cívicos, ha de ser más firme y decidida para proseguir esta evolución.

Este objetivo está alcanzando ya un grado tal de madurez que permmite reformular las medidas orientadas a una cierta infancia que está sometida a condiciones particulares de riesgo, así como renovar su organización y sus métodos con el fin de adaptarse a las nuevas necesidades de los niños y a la situación actual de los sistemas del bienestar. 
La presente ley tiene por objeto consolidar esta política integradora, preventiva, compensadora y de sensibilización cívica y social a través de los mecanismos de planificación, programación y evaluación conjunta entre todas las administraciones públicas y las instituciones sociales, que tendrá como eje el Plan Integral de Atención a la Infancia.

A la exigencia de renovación inherente a cualquier sistema, los dispositivos de atención a la infancia en situación de riesgo, desamparo e inadaptación han de responder a una triple demanda: a) atender a las transformaciones de los riesgos que pesan sobre la condición del niño, b) garantizar una oferta de calidad y una promoción de oportunidades para la infancia desfavorecida, y c) configurar una ordenación integrada y coherente de los diferentes sistemas administrativos que se ocupan del niño.

La reforma del sistema de atención a la infancia viene exigida, además, por la necesidad de desarrollar los requerimientos de la Ley Orgánica 4/1992, de 5 de junio, sobre la Reforma de la Ley Reguladora de la Competencia y del Procedimiento de los Juzgados de Menores, cuyas resoluciones son ejecutadas en el ámbito valenciano por la Generalitat Valenciana.

2. Existe hoy un fuerte apremio social ante el crecimiento de los riesgos y el aumento de la inadaptación de la infancia, que se traduce en una demanda sostenida de mayores y diferentes mecanismos protectores, como también la potenciación de los recursos personales y familiares.

A la tradicional problemática del abandono familiar, que generó unas respuestas centradas básicamente sobre la adopción y el hospicio, se añade hoy un amplio elenco de riesgos que cristaliza en determinados fenómenos sociales, como la violencia sobre el y la menor, el abandono escolar, la huida familiar, la explotación sexual, el uso indebido de drogas y la utilización de la imagen del menor.

Actualmente se exige proteger a la niña y al niño con medios apropiados frente a los agentes externos y los riesgos generados por las sociedades avanzadas que atenten contra la natural vulnerabilidad de aquél y que sean un peligro para la formación integral. En la medida en que los riesgos están diseminados por todo el cuerpo social y afectan a toda la población infantil, ya no es posible que las administraciones solas puedan prevenir los riesgos y aminorarlos. En su lugar se impone un compromiso conjunto de la sociedad y de las administraciones. Finalmente, en la medida en que la inadaptación de la infancia y de la juventud es un proceso multicasual ${ }^{2}$, ya no es posible judicializar las

2 Textualmente «multicasual» en el DOGV de referencia, aunque debe entenderse por el contexto como «multicausal» (Nota del Coordinador) 
medidas. En su lugar se impone la colaboración entre los órganos jurisdiccionales, la policía, las administraciones públicas y los agentes sociales, con una gran contribución de los medios de comunicación.

3. Si la infancia es uno de los intereses públicos más preciados en las sociedades modernas, su atención se ha convertido en un servicio público esencial en los países que se han constituido en un estado social y democrático de derecho. Es necesaria una política activa de defensa y de prevención que complete las modalidades tradicionales de asistencia y reeducación.

Con el fin de dar coherencia a las distintas modalidades de intervención, la ley organiza los programas en torno a dos niveles fundamentales: el nivel primario de carácter general que se dirige a toda la población infantil y el nivel especializado de alto contenido técnico y profesional que se dirige a los niños con necesidades sociales y familiares específicas.

La presente ley establece los distintos niveles de intervención mediante un diseño claro de responsabilidades de cada administración pública. Se propone así identificar las competencias de cada administración atendiendo a criterios de pertinencia, eficacia y coherencia y no de autoexclusión o de elusión de responsabilidad. De este modo se atiende a una de las mayores demandas que solicita mecanismos de coordinación de las políticas sectoriales como consecuencia de las interacciones de la problemática que afecta a los niños con necesidades especiales.

4. Las nuevas necesidades de los niños, la calidad de los servicios a la infancia y la promoción de nuevas oportunidades requieren una articulación adecuada de las administraciones educativas, sanitarias, sociales, culturales y de preparación al mundo laboral. La política social a favor de la infancia no necesita tanto una ampliación de recursos, cuanto una armonización, delimitación y coordinación de las políticas existentes.

Esta armonización de las políticas de infancia es la clave de bóveda de la presente Ley. Sólo de este modo es posible abordar la problemática del desamparo y de la inadaptación de las y de los menores que tiene en las sociedades avanzadas un carácter transversal que se disemina a través de todos los sistemas del bienestar. Sólo a través de un sistema cohesionado y ordenado es posible atender las nuevas necesidades de la infancia.

La presente Ley intenta situar la política compensatoria que desarrolla los servicios sociales en el interior de los sistemas ordinarios así como promover y reforzar la coordinación entre los distintos departamentos. La renovación de los mecanismos de protección y la promo- 
ción de las oportunidades para la infancia se torna inseparable de la cohesión interna del sistema y de la participación de la sociedad en la prevención de los riesgos y en la inserción social. Sistema que se concibe en la ley bajo una óptica global, que reúne en un mismo núcleo a todos los agentes titulares de funciones, deberes y potestades en la atención a los niños y las niñas. Sólo desde la nueva perspectiva que incorpora la ley son asumibles ámbitos, hasta hace poco ignorados, como la imagen del y de la menor en los medios de comunicación o la regulación del consumo de sustancias nocivas para la salud infantil, en los que el bienestar físico y psíquico de los niños y niñas y la satisfacción de sus necesidades aparecen directamente vinculados, pero donde las responsabilidades se diluyen entre las instituciones públicas, los agentes sociales y las propias unidades de convivencia. Y es, por ello, el propósito de la Ley abordar esa actuación global desde el prisma de los sistemas ordinarios de protección social como fórmula de garantía de la continuidad, interactividad y eficacia de tales sistemas, bajo el sistema de responsabilidad social general.

5. La presente Ley supone el desarrollo de las competencias que, en orden a la promoción y defensa de la y del menor le atribuye a la Generalitat Valenciana el artículo treinta y uno, apartado veintisiete, de Estatuto de Autonomía, e introduce un principio de ordenación estructural de la pluralidad normativa que, desde perspectivas parciales, propiciaban el incremento de los niveles de bienestar de la infancia. La Ley tiene, en este sentido, un carácter primordialmente social, de acuerdo con las recientes orientaciones de la ,legislación internacional, estatal y autonómica. No pretende regular un estatuto jurídico de la y del menor, sino que incorpora los derechos individuales y colectivos de los niños y niñas reconocidos constitucionalmente, por instrumentos internacionales o en la legislación civil estatal, que vienen a conformar el entramado jurídico garantista, especialmente en los que respecta a la Convención de las Naciones Unidas en 1989, así como la reforma del Código Civil operada por Ley 21/1987, de 11 de noviembre, lo que permite enfatizar el carácter ordenador e integrador de la Ley.

\section{TÍTULO PRELIMINAR}

\section{CAPÍTULO I}

Principios generales

Artículo primero. Objeto de la Ley.

La presente Ley tiene por objeto regular de forma integral la actuación de las instituciones públicas valencianas, los agentes sociales y 
los ciudadanos, en orden a procurar la atención e integración de los niños y las niñas en todos los ámbitos de convivencia. Se entiende por niño y niña, a los efectos de esta ley, todo menor de edad.

Artículo segundo. Situación de riesgo, desamparo e inadaptación.

Se considera situación de riesgo aquélla en la que por sus circunstancias personales o por influencias de su entorno o extrañas, exijan la adopción de medidas de prevención y rehabilitación para evitar situaciones de desamparo o de inadaptación.

Se considera situación de desamparo, conforme al artículo 172 del Código Civil, la que se produce de hecho a causa del incumplimiento, o del imposible o inadecuado ejercicio de los deberes de protección establecidos por las leyes para la guarda de las y los menores, cuando éstos queden privados de la necesaria asistencia ética, moral y material, y sea necesaria la adopción de medidas de protección y defensa.

Se considera situación de inadaptación aquélla que es declarada mediante resolución judicial, en los términos previstos por la Ley Orgánica de Reforma de la Ley Reguladora de la Competencia y el Procedimiento de los Juzgados de Menores, y que exige la adopción de medidas de resocialización e inserción.

\section{Artículo tercero. Principios rectores.}

La protección integral de la infancia, la prevención de los riesgos y la defensa y garantía de sus derechos reconocidos por la Constitución y por los acuerdos internacionales que velan por su efectividad, constituyen una responsabilidad indeclinable de todos los agentes que intervienen en la atención a la infancia y son los principios rectores de la actuación de los poderes públicos y de las relaciones del adulto con el niño.

\section{CAPÍTULO II}

Los agentes de la atención a la infancia

\section{Artículo cuarto. La Generalitat Valenciana}

El Gobierno Valenciano pondrá a disposición de las acciones que regula esta ley los programas adscritos a los servicios sociales especializados por la Ley 5/1989, de 6 de julio, y asumirá la coordinación general de la atención a la infancia, la planificación general de la misma y la evaluación de los programas.

Igualmente, los recursos de los sistemas sanitario y educativo serán puestos a disposición de los programas que se regulan en la presente Ley y de los que se sigan en el futuro. 
Artículo quinto. Las corporaciones locales.

Las administraciones locales, en el marco de la legislación vigente y a través de sus servicios sociales realizarán, en el ámbito de su competencia, las funciones de prevención, información, promoción y reinserción social en materia de menores, y colaborarán con la Generalitat Valenciana en la orientación y seguimiento de los casos que requieran su intervención en el propio medio. La Generalitat Valenciana prestará la necesaria cooperación técnica y financiera para el efectivo cumplimiento de estas funciones, atendiendo fundamentalmente al nivel de asunción por la correspondiente corporación local de las funciones que se regulan en el título segundo de esta ley.

Artículo sexto. Unidades de convivencia.

Los hijos no emancipados están bajo la potestad del padre y de la madre, quienes tendrán los deberes y facultades que les son propios según el artículo 154 del Código Civil.

Los padres y las madres deben prestar a los hijos habidos dentro o fuera del matrimonio asistencia de todo orden, durante su minoría de edad y en los demás casos que legalmente proceda.

Los deberes y funciones a que se refiere el párrafo anterior serán igualmente aplicables a las unidades de convivencia que se regulan en el capítulo IV del título segundo, tales como familias educadoras, familias de acogida y centros de residencia del niño, con sujeción, en este último caso a lo que disponga el Estatuto de Centros de Atención a la Infancia y Juventud y a los principios de esta ley.

Artículo séptimo. Instituciones de integración familiar.

Tendrán la consideración de instituciones colaboradoras de integración familiar, las asociaciones y fundaciones sin ánimo de lucro constituidas conforme a las leyes aplicables que obtengan la correspondiente acreditación en los términos establecidos reglamentariamente y figuren inscritas en el Registro constituido al efecto, sin cuyos requisitos no podrán ser consideradas como tales.

Artículo octavo. La infancia.

Los niños y niñas, en cuanto sus condiciones de madurez lo permitan, deberán participar activamente en las actividades que se realicen en su núcleo primario de convivencia y en todo aquello que le concierna, procurándose su plena integración en la vida familiar y social.

Para el logro de estos fines, la Generalitat Valenciana desarrollará programas dirigidos a promover:

a) El conocimiento y fomento de los recursos destinados a la atención de la infancia.

b) La sensibilización de la infancia en los valores democráticos. 
c) La creación de lugares de esparcimiento y encuentro.

d) El desarrollo cultural de la infancia.

e) El fomento del asociacionismo.

f) El ajuste de los recursos y núcleos de convivencia a la individualidad del niño y su grupo cercano.

g) La creación de condiciones ambientales que propicien el rechazo de la violencia en todas sus expresiones.

Artículo noveno. Principio de atención continua.

La Generalitat Valenciana garantizará la existencia de un sistema permanente de atención inmediato al niño, que permita atender situaciones de urgencia.

Se asegurará el conocimiento general de este recurso y su forma de acceso.

\section{TÍTULO PRIMERO}

La planificación

Artículo diez. El Plan Integral de Atención a la Infancia.

En el marco de la planificación general, el Gobierno Valenciano aprobará un Plan Integral de Atención a la Infancia, cuyo procedimiento de elaboración y aprobación se regirá por lo preceptuado en este título.

El Plan Integral de Atención a la Infancia será observado y complementado en la planificación sectorial, especialmente en los ámbitos social, cultural, educativo, sanitario y de preparación al mundo laboral.

El Plan de Atención al y la menor se elaborará en coordinación entre los diferentes órganos competentes de las diferentes consellerías afectadas, en colaboración con las entidades ciudadanas apropiadas (organizaciones de voluntariado, representación familias acogida, instituciones de acogida, asociaciones de vecinos).

\section{CAPÍTULO I}

Objeto y ámbito de la planificación

Artículo once. Necesidades y recursos

La planificación tendrá como objetivo general, en cada momento, satisfacer las necesidades del niño y la niña de acuerdo con su edad y circunstancias con el fin de lograr un mejor desarrollo y equilibrio personal.

Cada administración, en el ámbito de sus competencias, elaborará instrumentos para la detección de las necesidades a través de sus sistemas de atención primaria. 
Artículo doce. Ámbito temporal y territorial de la planificación.

$\mathrm{El}$ instrumento de planificación tendrá vigencia durante tres ejercicios presupuestarios, computados desde el siguiente a aquél en que se apruebe, sin perjuicio de su prórroga cuando así se acuerde. Durante la vigencia de cada plan, podrá ser modificado o revisado para su adaptación a las nuevas circunstancias.

La planificación de la atención a la infancia será única para todo el territorio de la Comunidad Valenciana. Sus determinaciones serán desarrolladas territorialmente en demarcaciones homologadas por la Generalitat Valenciana que agrupen a municipios cuyas circunstancias socio-económicas, de población, tipología familiar y de dotación de recursos exijan una actuación homogénea.

\section{CAPÍTULO II}

Elaboración y aprobación de la planificación

Artículo trece. Aprobación de la planificación.

Cada una de las consellerías competentes, conforme a lo establecido en el capítulo I anterior, elaborará una propuesta de la planificación, a la cual se acompañará:

a) Memoria explicativa del contenido del plan.

b) Previsión de resultados, junto a la evaluación de las necesidades.

c) Proyección presupuestaria del plan.

d) Informes, estudios, dictámenes y consultas realizados con carácter previo para la elaboración de la planificación.

El Gobierno Valenciano, a propuesta del Conseller de Trabajo y Asuntos Sociales, aprobará el Plan Integral de Atención a la Infancia. Igual procedimiento se seguirá para la modificación y revisión de la planificación.

El Plan será público y vinculará a la Generalitat Valenciana, y no creará por sí solo, derechos en favor de los particulares.

Artículo catorce. Contenido de la planificación.

El Plan Integral de Atención a la Infancia deberá contener, necesariamente:

a) Relación de las demandas generales o específicas existentes y previsibles.

b) Inventario de recursos adscritos a la atención a la infancia.

c) Características básicas de los recursos.

d) Asignación de recursos para las demandas actual y futura.

e) Programas de actuación durante el período de la planificación, conforme a lo que se establece en el título segundo.

f) Infraestructura básica de los programas. 
g) Adscripción de recursos a los programas.

h) Priorización y compatibilidad de los programas y la coordinación con los restantes programas de bienestar social;

i) Necesidades financieras derivadas de la planificación.

\section{TÍTULO SEGUNDO}

Programas, recursos y equipamientos

Artículo quince. Programas de atención al niño.

Con el fin de implantar las medidas de prevención y defensa, asistencia y rehabilitación, y resocialización e inserción, el Plan Integral de Atención a la Infancia incluirá preceptivamente el desarrollo de los siguientes programas: información, accesibilidad, cooperación, convivencia y reinserción social.

Las medidas de atención primaria serán competencia municipal, siendo coordinadas por los servicios sociales generales en el ámbito territorial del municipio, y desarrolladas anualmente mediante los instrumentos de colaboración que se establezcan. La evaluación de las medidas tendrá carácter anual, a cargo conjuntamente del Gobierno Valenciano y del ayuntamiento respectivo.

Los servicios especializados serán competencia de la Generalitat Valenciana y de los ayuntamientos, en los términos que recoge la Ley de Servicios Sociales de la Comunidad Valenciana, siendo coordinados por el órgano competente en materia de servicios sociales, y su evaluación tendrá carácter anual.

\section{CAPÍTULO I}

Programa de información

Artículo dieciséis. Campañas informativas y servicios de asesoramiento y primer diagnóstico.

Los ayuntamientos implantarán mediante los programas de prestaciones básicas de servicios sociales en las corporaciones locales un programa de información para las situaciones de riesgo, desamparo e inadaptación de niños, niñas y jóvenes, que contemplará como mínimo las siguientes medidas:

- Campañas informativas y de sensibilización.

- Servicios de asesoramiento y/o primer diagnóstico.

Artículo diecisiete. Campañas autonómicas y servicios de diagnóstico especializado.

El Gobierno valenciano implantará a través de sus respectivos de- 
partamentos la creación y desarrollo de medidas de información con carácter especializado y establecerá, al menos, los siguientes recursos:

- campañas autonómicas.

- servicios de diagnóstico especializado.

La Generalitat Valenciana llevará a cabo campañas específicas contra el uso de la violencia en el medio infantil y juvenil, y evitará la emisión de cualquier programa audiovisual en los medios de comunicación públicos, especialmente TVV, y promocionará los video-juegos que tengan un carácter contrario a la violencia.

La Generalitat Valenciana realizará campañas en el ámbito infantil y juvenil encaminadas a combatir las actitudes racistas y sexistas, que mientras tanto se dan en la sociedad, con la finalidad de contribuir a que ésta sea cada vez más tolerante e igualitaria.

\section{CAPÍTULO SEGUNDO}

Programas de accesibilidad

Artículo dieciocho. Absentismo escolar, salud infantil e inserción prelaboral.

Los ayuntamientos implantarán un programa de accesibilidad dirigido a niños y jóvenes con el objeto de mantenerles en su medio natural y favorece su incorporación a la sociedad, y contemplará, al menos, las siguientes modalidades:

- Seguimiento del absentismo escolar.

- Promoción de la salud infantil.

- Fomento de la inserción prelaboral.

Artículo diecinueve. Prevención de malos tratos e integración escolar y laboral y eliminación de barreras.

El Gobierno Valenciano implantará, a través de sus respectivas consellerías, la creación y desarrollo de los servicios especializados de accesibilidad que requieran un alto contenido técnico y profesional, y establecerá, al menos, los siguientes programas:

- Prevención de los malos tratos.

- Integración escolar.

- Medidas laborales de inserción.

- Eliminación de barreras arquitectónicas y de comunicación.

Los órganos competentes de la Generalitat Valenciana adoptarán las medidas necesarias para remover barreras arquitectónicas, de comunicación y cuantos obstáculos impidan la plena integración de la infancia en la vida social y cultural, con una especial sensibilidad hacia aquellos o aquellas menores con minusvalías físicas o psíquicas. 


\section{CAPÍTULO III}

Programas de cooperación

Artículo veinte.Promoción de la cooperación social.

Los ayuntamientos implantarán un programa de cooperación con el fin de fomentar las organizaciones solidarias y el voluntariado en el ámbito de la atención a la infancia y juventud que contemplará como mínimo las siguientes medidas:

- Promoción de la auto-ayuda.

- Fomento del voluntariado social.

- Promoción de las organizaciones solidarias.

Artículo veintiuno. Ordenación de la cooperación social.

El Gobierno Valenciano completará la red pública de atención a la infancia mediante el establecimiento de vías de cooperación con servicios especializados que requieran mayor contenido técnico y se consideren necesarios dentro de la planificación general.

Con este fin, el Gobierno Valenciano desarrollará, al menos, las siguientes actuaciones:

a) Acreditación de centros y servicios.

b) Regulación del régimen de cooperación.

c) Reconocimiento de las instituciones colaboradoras de integración familiar.

\section{CAPÍTULO IV}

\section{Programas de convivencia}

Artículo veintidós. La guarda y la tutela de menores.

El programa de convivencia tiene por finalidad el mantenimiento del niño y la niña en su propia familia y la integración transitoria o definitiva en otros núcleos de convivencia que favorezcan su desarrollo integral. La Generalitat Valenciana asumirá la guarda y tutela de menores, en las condiciones siguientes:

1. La guarda se ejercerá a solicitud de quienes tienen la potestad sobre el menor por causas graves justificadas, o por acuerdo del juez en los casos que legalmente proceda. La no asunción de la guarda por la administración deberá ser motivada, y cesará de oficio o a instancia de parte por finalización de la causa que lo motivó.

La guarda será ejercida por la Generalitat Valenciana, por los ayuntamientos y por instituciones de integración familiar, debidamente acreditadas y coordinadas.

2. La Generalitat Valenciana ejercerá la tutela de los menores en situación de desamparo prevista en el artículo 172.1 del Código Civil. 
La apreciación de la situación de desamparo, bien de oficio o a instancia de parte se hará por resolución administrativa que se notificará a los titulares de la patria potestad con indicación de la medida y al ministerio Fiscal.

El cese de la tutela que se constituye por ministerio de la ley, se producirá por desaparición de las circunstancia que las motivaron, por la adopción del o la menor, por la emancipación o mayoría de edad del tutelado, por el fallecimiento del tutelado o por la constitución de la tutela ordinaria.

3. Se exceptúa de lo dispuesto en este artículo las medidas incluidas en el siguiente.

Artículo veintitrés. La ayuda familiar.

Los ayuntamientos implantarán un programa de convivencia con el fin de favorecer la integración del niño en su propia familia y fomentar recursos de guarda, a través de las modalidades que reglamentariamente se determinen.

La Generalitat Valenciana en colaboración con los ayuntamientos dará prioridad a las ayudas que tengan por objeto satisfacer necesidades básicas del niño y la niña, a través del sistema público de servicios sociales, a fin de prevenir la generación de situaciones de desamparo, y en los casos en que cualquiera de los progenitores incumple los deberes impuestos normativa o judicialmente.

Artículo veinticuatro. Acogimiento de carácter primario.

El acogimiento familiar a nivel primario será desarrollado por los equipos sociales de base y podrá realizarse mediante la simple guarda en su familia extensa o en el vecindario, o a través de una familia educadora.

Artículo veinticinco. Atención especializada a la convivencia.

El Gobierno Valenciano desarrollará los servicios especializados de convivencia que requieran un alto contenido técnico y profesional con el fin de arbitrar las alternativas a las situaciones de desamparo y al conflicto familiar grave. El programa de convivencia contemplará como servicios especializados, al menos, los siguientes recursos:

- La terapia familiar dirigida a aplicar a una unidad de convivencia prestaciones técnicas que permitan abordar los conflictos relacionales y la desestructuración familiar.

- El acogimiento de carácter especializado, ya se produzca en la familia extensa, en un núcleo familiar ajeno al menor o a la menor o en una residencia.

- La propuesta de adopción ante el órgano judicial competente. 
Artículo veintiséis. Acogimiento de carácter especializado.

$\mathrm{El}$ acogimiento familiar de carácter especializado produce la integración de un niño o una niña en otra unidad de convivencia por decisión administrativa o judicial cuando concurren especiales dificultades en el o la menor, o falta de consentimiento de la familia de origen. Si entre el niño o la niña y la unidad de convivencia en que se integra no existieren vínculos parentales, el acogimiento puede revestir una de estas dos formas: familia educadora y acogimiento preadoptivo.

Se aplicará el recurso de la familia educadora cuando sea posible realizar un proyecto educativo entre la familia biológica y la acogedora, facilitando la reinserción del niño o la niña en su familia de origen y removiendo los obstáculos que lo impiden.

El acogimiento preadoptivo tiene por objeto la integración de un niño o una niña en un núcleo de convivencia como paso previo a la formalización de la adopción, con el fin de garantizar la idoneidad de la medida.

Artículo veintisiete. Acogimiento en residencia.

El acogimiento en residencia se realizará mediante la guarda del menor o de la menor en un centro de protección, siempre que las circunstancias del caso no hicieran aconsejable adoptar alguna de las medidas reguladas en los artículos anteriores.

La Generalitat Valenciana dispondrá de residencias comarcales, infantiles y juveniles, propias o concertadas, dotadas suficientemente y que permitan la plena aplicación de lo dispuesto en este artículo.

Artículo veintiocho. Adopción de menores.

La adopción se regirá, en cuanto a su constitución y efectos, por lo que dispone la legislación civil del Estado. La propuesta previa, que en su caso, deba realizar la Generalitat Valenciana será formulada por el Consejo de Adopción, cuya organización y funciones se desarrollarán reglamentariamente atendiendo al supremo interés del niño y la niña. No será en ningún caso considerada una medida discriminatoria para conceder una adopción, el tipo de núcleo de convivencia familiar por el que hayan optado libremente aquellos o aquellas que soliciten la adopción.

Con carácter previo a la formalización ante el Juez de la adopción, en los casos de acogimiento preadoptivo, deberá existir un período de convivencia entre el o la menor y los posibles adoptantes, como garantía de su idoneidad. 


\section{CAPÍTULO V \\ Programas de reinserción}

Artículo veintinueve. Atención primaria de reinserción.

La Generalitat Valenciana en colaboración con los ayuntamientos implantarán el programa de reinserción social dirigido a menores en situación de inadaptación con el objeto de lograr la resocialización familiar y social y contemplará a nivel primario las siguientes medidas, que serán impuestas por resolución judicial:

- La amonestación.

- La libertad vigilada.

- El asesoramiento educativo, terapeútico y ocupảcional.

- La prestación de servicios en beneficio de la comunidad.

- El internamiento de uno a tres fines de semana, que se sujetará a lo dispuesto en el programa de convivencia.

- La reparación extrajudicial.

En el desarrollo de estos programas podrán colaborar las instituciones sociales que cuenten con centros o servicios acreditados para ello, en las condiciones que reglamentariamente se determinen.

Artículo treinta. Alternativas de ejecución a nivel primario.

Los servicios sociales generales pondrán a disposición de los Juzgados de Menores, como medidas alternativas o como instrumentos para la ejecución de la resolución judicial en virtud de la disposición adicional tercera de la Ley Orgánica 4/1992, de 5 de junio, sobre reforma de la Ley Reguladora de la Competencia y el Procedimiento de los Juzgados de Menores, los siguientes recursos:

- La instrucción de apoyo, que consistirá en el compromiso voluntario del o de la menor para recibir ayuda y seguir las instrucciones que, a título de tutoría social, se vayan valorando como positivas en relación a la situación del mismo.

- La instrucción de tratamiento terapéutico, que consistirá en el compromiso voluntario del o de la menor y, en su caso, de su familia, de acudir a un tratamiento con profesionales, una vez detectadas carencias relevantes en el ámbito familiar o individual.

- El asesoramiento educativo, que consistirá en el compromiso voluntario del o de la menor y familia de acudir a un programa educativo, en supuestos próximos al desamparo.

Artículo treinta y uno. Tratamiento ambulatorio y centros terapéuticos.

La Generalitat Valenciana implantará, de conformidad con la Ley Orgánica 4/1992, de 5 de junio, sobre Reforma de la Ley Reguladora de la Competencia y el Procedimiento de los Juzgados de Menores, los servicios especializados de reinserción necesarios para el cumplimiento 
de las resoluciones de los Juzgados de Menores mediante los siguientes recursos:

- El acogimiento por otra persona o núcleo familiar, que se sujetará a lo dispuesto en el programa de convivencia.

- El tratamiento ambulatorio.

- El ingreso en un centro de carácter terapéutico.

- El ingreso en un centro en régimen abierto, semi-abierto o cerrado.

El tratamiento ambulatorio dispondrá de los servicios técnicos de carácter educativo y sanitario que se ofrecen al menor en régimen abierto mediante los centros de salud, las unidades de salud mental, centros de día y cuantos recursos se habiliten para ello en la red ordinaria.

$\mathrm{El}$ ingreso en un centro de carácter terapéutico se realizará en aquellos centros residenciales que ofrecen tratamiento especializado de carácter educativo y sanitario a través de la red ordinaria, tales como comunidades terapéuticas, centros de día, unidades hospitalarias y cuantos recursos se habiliten para ello.

Artículo treinta y dos. Internamiento en régimen abierto.

$\mathrm{El}$ internamiento en régimen abierto comprende la obligación de residir en un centro de carácter socioeducativo que desarrolle hábitos de convivencia y apoyo sicosocial en interacción con el medio donde esté ubicado. Las actividades escolares se realizarán fuera del mismo y los y las jóvenes sujetos a esta medida disfrutarán de fines de semana y de vacaciones en sus propios hogares.

Las medidas de ingreso en un centro de régimen abierto acordadas por los juzgados de menores podrán ejecutarse en las Residencias comarcales, en las residencias juveniles, en los centros de recepción y en cuantos recursos se habiliten para ello.

Articulo treinta y tres. Internamiento en régimen semiabierto.

El internamiento en régimen semiabierto consiste en la obligación de residir en un centro de carácter socioeducativo que desarrolle hábitos de convivencia, actividades rehabilitadoras y apoyo sicosocial en interacción controlada con el medio donde esté ubicado. Las actividades escolares y preläborales se realizarán como norma general dentro del centro, excepto en los casos que se justifique lo contrario. Los y las jóvenes sujetos a esta medida podrán realizar actividades extraescolares fuera del centro y disfrutar de fines de semana y vacaciones.

Las medidas de ingreso en un centro en régimen semiabierto acordadas por los juzgados de menores podrán ejecutarse en los centros de recepción, de reeducación, en residencias comarcales y en cuantos recursos se habiliten para ello de acuerdo con el reglamento de régimen interior del centro. 
Artículo treinta y cuatro. Internamiento en régimen cerrado.

$\mathrm{El}$ internamiento en régimen cerrado impone la obligación de residir en un centro de carácter socioeducativo que desarrolle hábitos de convivencia, actividades rehabilitadoras y poyo sicosocial en privación de libertad por resolución judicial. El régimen cerrado estará a cargo de un personal de especial provisión con formación específica, que garantizará la custodia y seguridad de las y de los menores así como su seguimiento individualizado. Las y los jóvenes sujetos a esta medida no podrán salir del centro sin autorización judicial, y cuando sea necesario lo harán acompañados de un educador del centro.

Las medidas de ingreso en un centro en régimen cerrado acordadas por los juzgados de menores podrán ejecutarse indistintamente en cualesquiera centros de reeducación de la Comunidad Valenciana o en las unidades de los mismos, adecuadamente preparadas, y en cuantos recursos se habiliten para ello.

Artículo treinta y cinco. Estatuto de centros.

Los regímenes abierto, semiabierto y cerrado, se regirán por el Estatuto de Centros. En todo caso con el fin de concluir el proceso educativo iniciado a partir de una medida judicial se asegurarán los apoyos educativos, asistenciales y prelaborales en orden a la reinserción social.

La evaluación del programa de reinserción se realizará anualmente a cargo del Gobierno Valenciano, en coordinación con cuantos otros órganos o instancias tengan encomendado el seguimiento de los y de las menores en situación de inadaptación.

Artículo treinta y seis. Principios sancionadores.

Las sanciones tendrán carácter individualizado, y para su imposición se tendrá en cuanta el grado de madurez del y de la menor y el grado de voluntariedad manifestado en su comisión.

En todo caso, quedan prohibidas las siguientes sanciones:

- Castigos corporales.

- Privación de alimentos.

- Privación de asistencia a la escuela.

- Privación del derecho de visita de su familia.

- Expulsión del centro, sin dar otras alternativas educativas.

- Aislamiento del y de la menor, salvo en casos excepcionales y con inmediata comunicación al juzgado correspondiente.

Artículo treinta y siete. Infracciones del régimen interno de los centros.

Las y los menores ingresados en un centro en régimen abierto, semiabierto o cerrado, quedarán sujetos a las normas de convivencia que se regulen en su reglamento de régimen interno. Las infracciones de dichas normas se calificarán como faltas, que podrán ser leves, graves y muy graves. 
Son faltas leves las que suponen un incumplimiento de las reglas básicas de organización del centro, tales como horarios, programas de actividades, distribución del espacio, falta de higiene, indebido uso de bienes y servicios, y cualquier otra infracción leve a las normas de convivencia.

Son faltas graves las ausencias del centro, causar daños al centro o a los bienes de un tercero, faltas de asistencia a la escuela y cualquier otra infracción que lesione el normal funcionamiento del centro o impida el cumplimiento de las medidas socio-educativas.

Son faltas muy graves las que supongan un atentado contra la persona o dignidad de los compañeros del centro o de los profesionales que desempeñen su servicio en ellos.

\section{Articulo treinta y ocho. Sanciones.}

Las faltas leves serán sancionadas con: amonestación, conciliación y disculpa ante los afectados, reparación del daño, multas económicas detraídas del dinero de bolsillo semanalmente asignado, reducción del tiempo de ocio.

Las faltas graves serán sancionadas con: restricción de salidas de la residencia, separación del grupo durante el tiempo libre, pérdida temporal de responsabilidades en el grupo. En ningún caso excederán tales sanciones del período de siete días.

Las faltas muy graves se sancionarán con: la pérdida definitiva de responsabilidades en el grupo, la no asignación de dinero de bolsillo durante el plazo que se fije, la restricción de salidas o tiempo libre por tiempo no superior a quince días.

Artículo treinta y nueve. Garantías procedimentales.

La imposición de sanciones por faltas graves y muy graves precisa la incoacción de expediente disciplinario con audiencia del inculpado y de la comisión educativa.

El director/a del centro será competente para imponer las sanciones correspondientes y para determinar la forma de ejecutarlas. Contra dichas resoluciones podrá interponerse un recurso administrativo ante la superioridad jerárquica del director/a, sin perjuicio de lo que establezcan las leyes procesales y de la superior vigilancia del Ministerio Fiscal.

\section{TÍTULO TERCERO}

Evaluación y control

Artículo cuarenta. Coordinación de los programas.

El órgano competente en materia de servicios sociales coordinará la ejecución de los programas de intervención que se regulan en el 
título segundo, sin perjuicio de la gestión que le corresponda respecto de aquellos programas que sean de su competencia.

La función de coordinación comprende la evaluación global de las acciones y la verificación de los resultados de la planificación.

Artículo cuarenta y uno. Seguimiento de los programas.

A la vista de los resultados de la evaluación se podrá proponer la modificación o adaptación del Plan de Atención Integral a la Infancia, formular recomendaciones a los órganos en cada caso competentes, adoptar las actuaciones que sean de su competencia e, incluso, proveer las medidas necesarias en situaciones de urgente necesidad social.

Para el cumplimiento de la finalidad establecida en el párrafo anterior, los órganos con competencia en servicios sociales que tengan atribuida la función de implantación de los programas regulados en esta ley se constituirán en observatorio permanente de la situación del niño en la Comunidad Valenciana, correspondiéndole la coordinación entre los órganos gestores y los órganos de planificación.

Artículo cuarenta y dos. Colaboración con la administración de justicia.

La Generalitat Valenciana informará al Ministerio Fiscal de la situación en que se encuentran las y los menores sujetos a tutela, guarda y acogimiento, los internamientos que se produzcan y la evolución y cese de las medidas, en cada caso, adoptadas.

Asimismo pondrá a disposición de los órganos jurisdiccionales sus equipos, centros y servicios para el desarrollo de sus funciones.

\section{DISPOSICIONES TRANSITORIAS}

\section{Primera}

Inmediatamente desde la publicación de esta ley, el Gobierno Valenciano aprobará el Reglamento General de la misma. Hasta que dicho reglamento no entre en vigor, regirán las normas de regulación de los recursos y medios y de los procedimientos para su aplicación vigentes actualmente, en cuanto no se opongan a las disposiciones de esta Ley.

\section{Segunda}

Inmediatamente, desde la publicación de esta Ley, el Gobierno Valenciano aprobará el Plan Integral de Atención a la Infancia regulado en el título primero de la misma.

\section{Tercera}

Los beneficios establecidos a favor de los niños y las niñas en la 
presente ley podrán ser de aplicación a las personas afectadas por una disminución psíquica, con independencia de su edad y en atención a sus necesidades específicas.

\section{DISPOSICIONES ADICIONALES}

\section{Primera}

Para el ejercicio efectivo de las competencias que el artículo 24 de la ley atribuye a los ayuntamientos en materia de acogimiento familiar de carácter primario,deberán suscribir previamente un convenio específico de colaboración con la Generalitat Valenciana en el que se regularán las condiciones para el ejercicio de estas funciones y la necesaria coordinación con la Generalitat Valenciana. En tanto no se suscriba dicho convenio, el acogimiento de carácter primario será ejercido por el órgano competente de la Generalitat Valenciana.

\section{Segunda}

La disposición anterior será igualmente aplicable para el efectivo ejercicio de las competencias que el artículo 29 atribuye a los ayuntamientos en materia de atención primaria de reinserción.

\section{Tercera}

Las funciones que esta Ley atribuye a los ayuntamientos, podrán ser ejecutadas por las mancomunidades o, en su caso, consorcios ya existentes o creados a tal efecto. En todo caso, en los estatutos de la entidad se expresará el órgano competente de la misma para resolver la aplicación de las medidas prevenidas en el título segundo de la Ley y, a falta de dicha expresión, el órgano de gobierno la atribuirá según las reglas de la institución.

\section{DISPOSICIÓN FINAL}

La presente Ley entrará en vigor a los seis meses de su publicación en el Diari Oficial de la Generalitat Valenciana, excepto las disposiciones de título preliminar y las contenidas en los artículos 37, 38, 39 y 40, que regirán desde el día de la publicación de la Ley. 